\title{
Developing the Metrics to Assess the Library's Active Learning Spaces
}

\author{
Karen R. Hum, Ningning Kong, Yue Li, and Nanette Andersson \\ Purdue University, USA
}

\begin{abstract}
Upon the opening of Purdue's new 179,000 square foot state-of-the-art Wilmeth Active Learning Center (WALC) facility, Purdue University Libraries (PUL) was interested in assessing space usage via observation to assist with the development of institutional metrics, as well as to ensure adequate staffing and services. Due to the size of the building and number of observations needed however, PUL team members determined that a more comprehensive data-collection tool was needed. The authors collaborated to design and build a mobile device-based, location-enabled system with reporting capabilities.

The data collection system was beta tested during the fall 2017 academic term. As part of this process, detailed training materials were provided to users to ensure data and technology consistency. Based on user feedback, the database and data collection interfaces were then modified to improve usability and data veracity. Results suggest the system is an effective tool for collecting library space usage information with minimal end-user training requirements. The system enables multiple staff members to collect consistent information in a reduced amount of time. Initial analyses have also helped to inform understanding of learning space usage statistics and the different learning activities occurring in the integrated areas, which will serve as a guideline for PUL's future service design.
\end{abstract}

\section{Purdue's Wilmeth Active Learning Center (WALC)}

Purdue University is a public land grant research institution located in West Lafayette, Indiana. Founded in 1869 , the university currently has more than 41,000 students enrolled and offers over 200 undergraduate majors and 69 post-graduate programs.

In August of 2017, Purdue opened the Thomas S. and Harvey D. Wilmeth Active Learning Center. The WALC is a new concept in university buildings and was envisioned to fully integrate library space with active learning instructional spaces (Figure 1). The 179,000 square-foot facility houses seven different types of active learning teaching spaces for a total of 27 classrooms on three floors plus a lower level. The classrooms offer state-of-the-art digital tools for facilitating collaboration, communication, and distance learning. 
Figure 1. The WALC layout
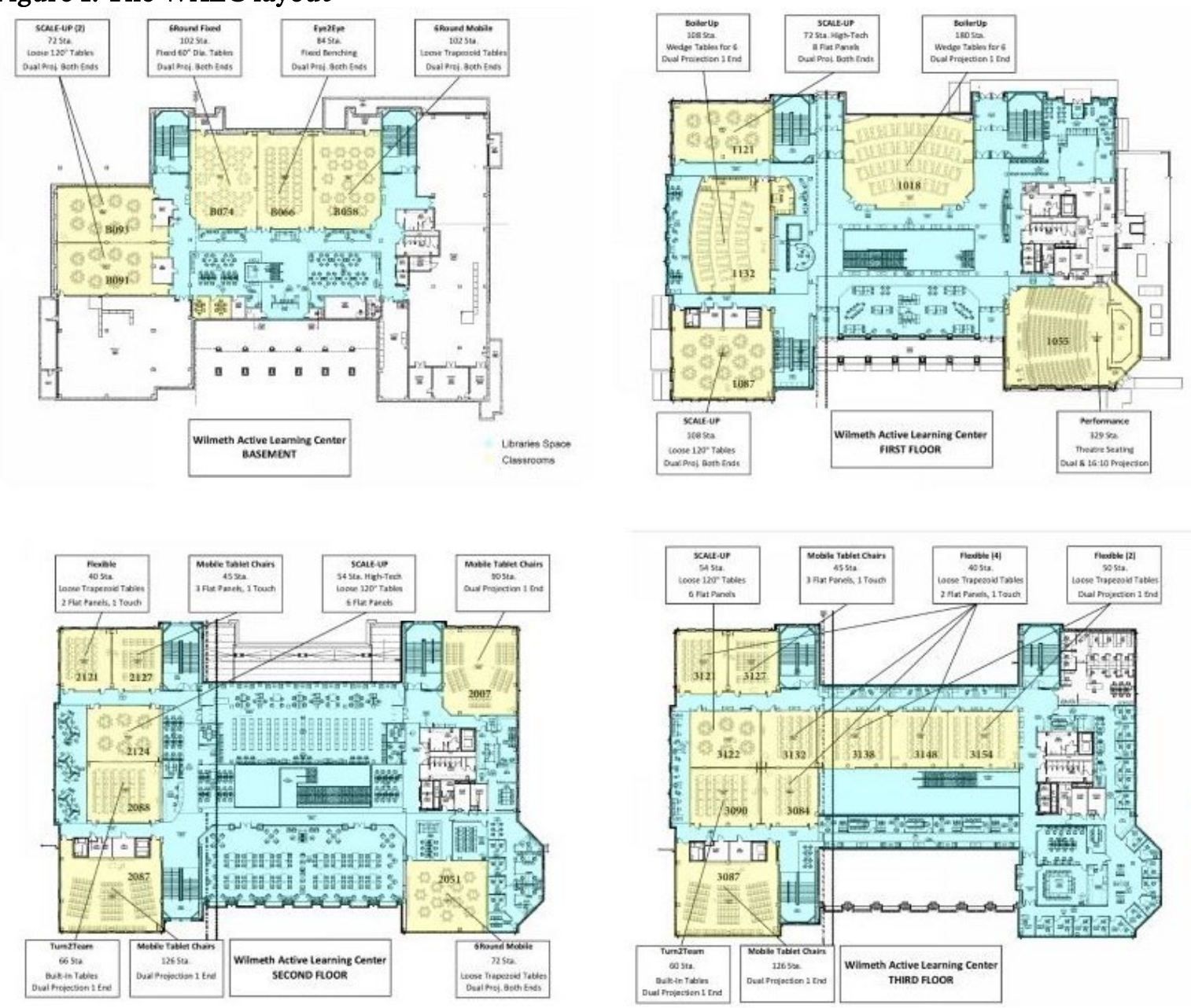

\section{LIBRARY CLASSROOM}

Surrounding the classrooms and interspersed throughout the building is Purdue University Libraries' Library of Engineering and Science (LOES). The library spaces include collection stacks with 30,000 volumes, study rooms, collaborative spaces, quiet study areas, and computer stations, as well as specialty computer labs and faculty offices. The library space is completely integrated with the classroom space, such that the collaborative library space is immediately adjacent to each of the classrooms. In addition, when classes are not being held, all of the classrooms are made available as additional study areas.

\section{Realizing the Need for a Comprehensive Data-Collection System}

The WALC's marriage of library space with classroom space evolved through a ten-year series of iterations of locating active learning classrooms in libraries. ${ }^{1}$ Due to the active-learning nature of the curriculum, students were divided into small groups to work collectively to solve problems. When the earlier versions of these active learning classrooms were placed among library spaces, two compelling outcomes were observed.

First, students from these courses often arrived in the library before class began to meet in their smaller groups and then stayed late after class, again spilling into the library spaces to continue working on the assigned problems. Their instructors would also frequently join them, thus extending the learning period. 
Several instructors even chose to hold their office hours in the library, where their students were already working, which appeared to result in more students taking advantage of office hours.

The second observation was that the juxtaposition of active learning classrooms with library spaces extended the use of the library. Typically, academic libraries are busy from 3:00 p.m. to 12:00 a.m., after most classes have been completed. Conversely, classrooms are busy from 7:00 a.m. to 3:00 p.m., as the majority of courses are scheduled during these hours. Holding active learning classes within the library spaces extended the "busy" hours from 7:00 a.m. through midnight, which effectively doubled use of the library spaces.

Because these observations were anecdotal, the decision was made when the WALC was constructed to closely monitor activity to determine if, in fact, the suspected increases in building usage and learning period were accurate. Purdue University Libraries' administration was also interested in assessing space usage via observation to assist with the development of institutional metrics, as well as to ensure adequate staffing and services. The WALC is staffed 24/7 during regular academic periods, so attendants were tasked with conducting observational head counts during their rounds.

At first, observations were recorded using paper and pencil, and the data were manually entered into an Excel spreadsheet at the end of each building attendant's shift. This quickly became problematic however, as it required both manual notation of each observation and the secondary step of data entry. Not only did this markedly inflate the amount of time needed to collect data, it provided repeated opportunities for data-entry errors to occur. In addition, during peak periods, staff were often distracted with other tasks, which raised concerns regarding missing and duplicate counts. Clearly, a more sophisticated method was needed.

\section{Development of an In-House Solution}

Similar to other academic institutions, Purdue University Libraries' (PUL) faculty and staff bring a wide variety of experience and expertise to the table. Thus, the development of a technical solution was quickly seen as an ideal opportunity for internal collaboration across specializations. PUL members joining the project were the director of assessment and the director of facilities (the assessment team), as well as a geographic information systems (GIS) analyst and an associate professor/GIS specialist (the GIS team).

Before committing to the enterprise, initial group discussions centered on whether more advanced options might already be available and if those options could be easily customized to meet required criteria. Two location-based library facility inventory tools were identified at this stage: Suma, developed by North Carolina State University Libraries, ${ }^{2}$ and the web GIS platform developed at the University of Idaho Library. ${ }^{3}$ A comparison study of the data collection requirements for our specific project was conducted against these systems, as was a review of all in-house resources toward supporting such a data collection system.

The user interface designed for Suma's mobile interface appeared intuitive enough to save significant staff training time, but it required a different technology stack than that currently existing within PUL. The map interface developed in the University of Idaho Library's system looked appealing because it provides a good reference for users as they move through a building, and its technology was also found to be compatible with PUL's existing infrastructure. However, because PUL anticipated expanded information collection related to different activities and preferred to save inventory data to a local database for further reporting and analysis, the GIS team determined that an in-house system would be PUL's best solution.

Concurrent with the GIS team's technical development of the system, ${ }^{4}$ the assessment team began reaching out to a variety of stakeholders-such as PUL administrators, division heads, and WALC building managersto identify key data points to be included. An important aspect of this step was to also uncover pending/wish list research projects so that any related data collection activities might be incorporated as well.

After a master list of variables was established, the assessment team worked to narrow down the field by clarifying variable definitions, consolidating highly similar data points, and eliminating those that would not 
lend themselves well to observation. The final list was designed to determine: (1) how the library spaces were being used by students at different times each day; (2) which library services and technology were being used by students and how they were being used; and 3) the types of activities students were engaging in while at the WALC.

This revised list of variables and definitions was next shared with the GIS team for incorporation into the data collection system, as were detailed WALC floor plans provided by the director of facilities. The two teams soon began a series of regular meetings to list system requirements, review progress, and suggest changes and additional features. Certain stakeholders were also invited to participate in some of these discussions so as to garner input from their perspective, and many of their recommendations were adopted as well.

The system was deemed ready for use during the fall 2017 academic term, so it was decided that, due to time constraints, beta testing would be done as part of a planned five-day data-collection initiative in the WALC to gain insight into building usage. At the same time, WALC building attendants also began to regularly use the system to collect observation data during their midnight to 8:00 a.m. shifts. To facilitate these endeavors, a detailed manual was developed and participating staff were provided with end-user training.

While the system worked as designed and the results provided valuable insights, user feedback indicated that a number of important modifications to the data-collection tool were needed. Subsequent system enhancements included mechanisms to monitor data-submission activity and a session number for improved data analysis. It was also determined that some areas of the WALC, including the lower level and near stairways, were experiencing Wi-Fi connection gaps, which sometimes affected data submission. The importance of this finding was two-fold, as Wi-Fi access problems could also impact student-learning activities.

A second large-scale data-collection project was conducted during the spring 2018 term and subsequent staff feedback suggested that the recent updates made to the system greatly improved the end-user experience. The changes also alleviated concerns regarding missing or duplicate data.

\section{Technical System Design}

The GIS team at PUL manages a set of GIS servers, including ArcGIS Servers, enterprise geodatabases, and ArcGIS Portal. These resources, which had already been used by many research groups on campus in terms of sharing maps and managing data, made a customized solution feasible.

The data collection system includes four major components: a mobile interface for data collection; a GIS server for centralized map and data sharing; a geodatabase with maps and tables; and an engine for data aggregation and reporting (Figure 2). The mobile interface was developed using JavaScript and ArcGIS API, supported by the map services published from ArcGIS Server. ${ }^{5}$ 
Figure 2. The system components

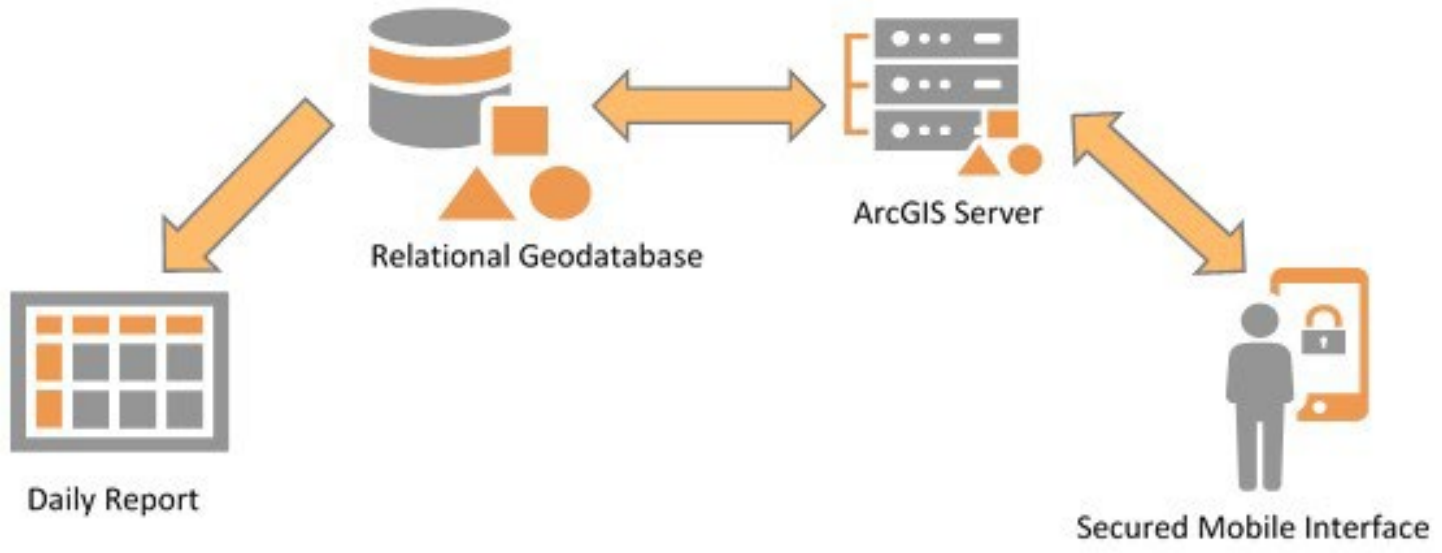

When developing the web interface, security and quality control were a major concern. The intent was that only authorized personnel would have access to the interface and be able to contribute data. At the same time, a record of who was contributing data was needed should substantially different observation estimates occur. This security feature was implemented using named users created for ArcGIS Portal. Passwordprotected user logins were established so that each observation would be associated with a specific user name, and observations could only be captured by the system via authorized users (Figure 3).

Figure 3. Secured login allows authorized personnel to input data.

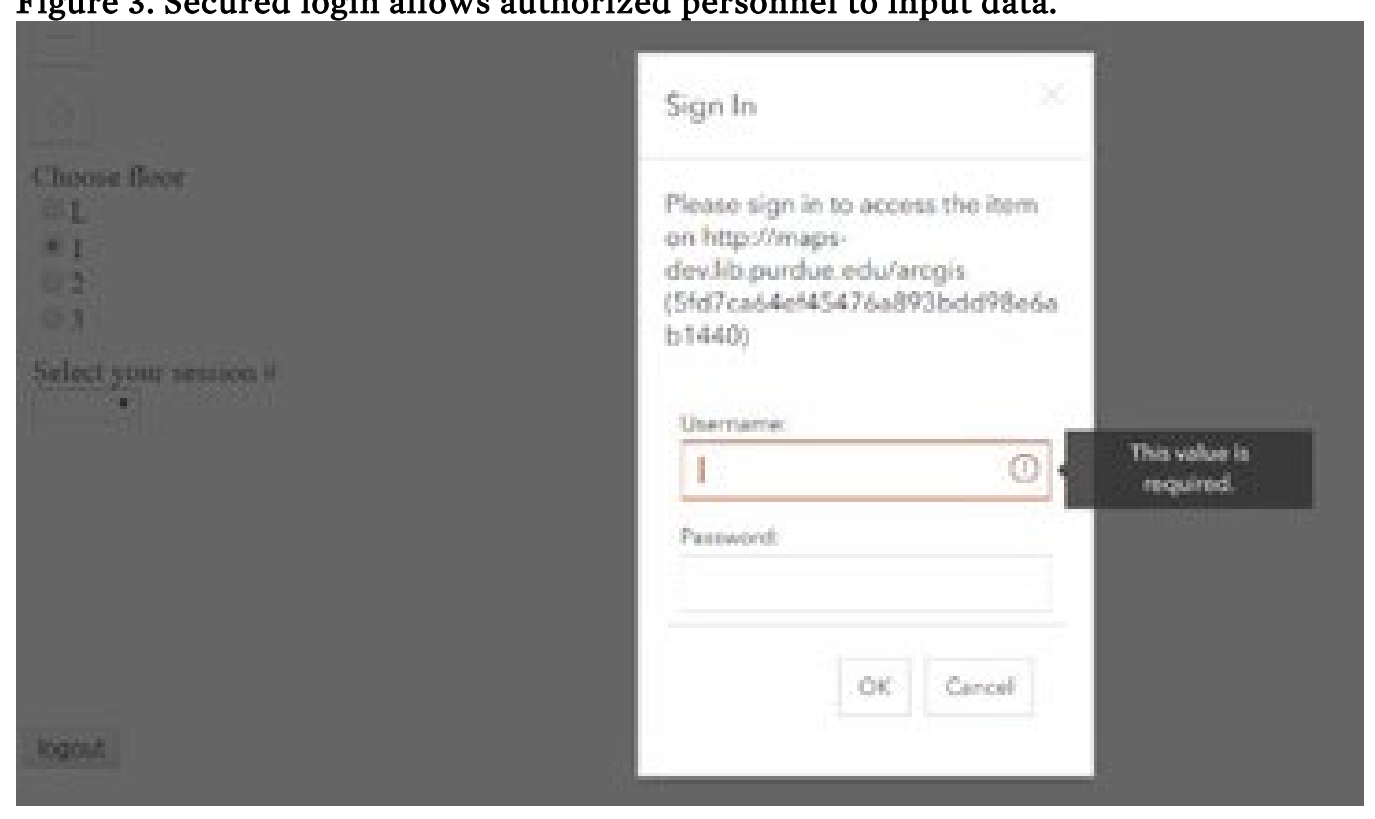

To develop the map interface, the WALC's building floor plans were used as reference maps to help users navigate while collecting data. After the scanned floor plans were georeferenced, all pertinent space areas were digitized and saved as feature classes in the geodatabase. To further enhance the data-collection process, the three distinctive types of areas identified on the maps were highlighted using different colors on the interface (Figure 4): (1) space open 24 hours per day, (2) classrooms closed at 2:00 a.m. daily, and (3) classrooms closed at midnight. These feature classes were then published as feature services through ArcGIS Server. 
Figure 4. The mobile data collection interface.

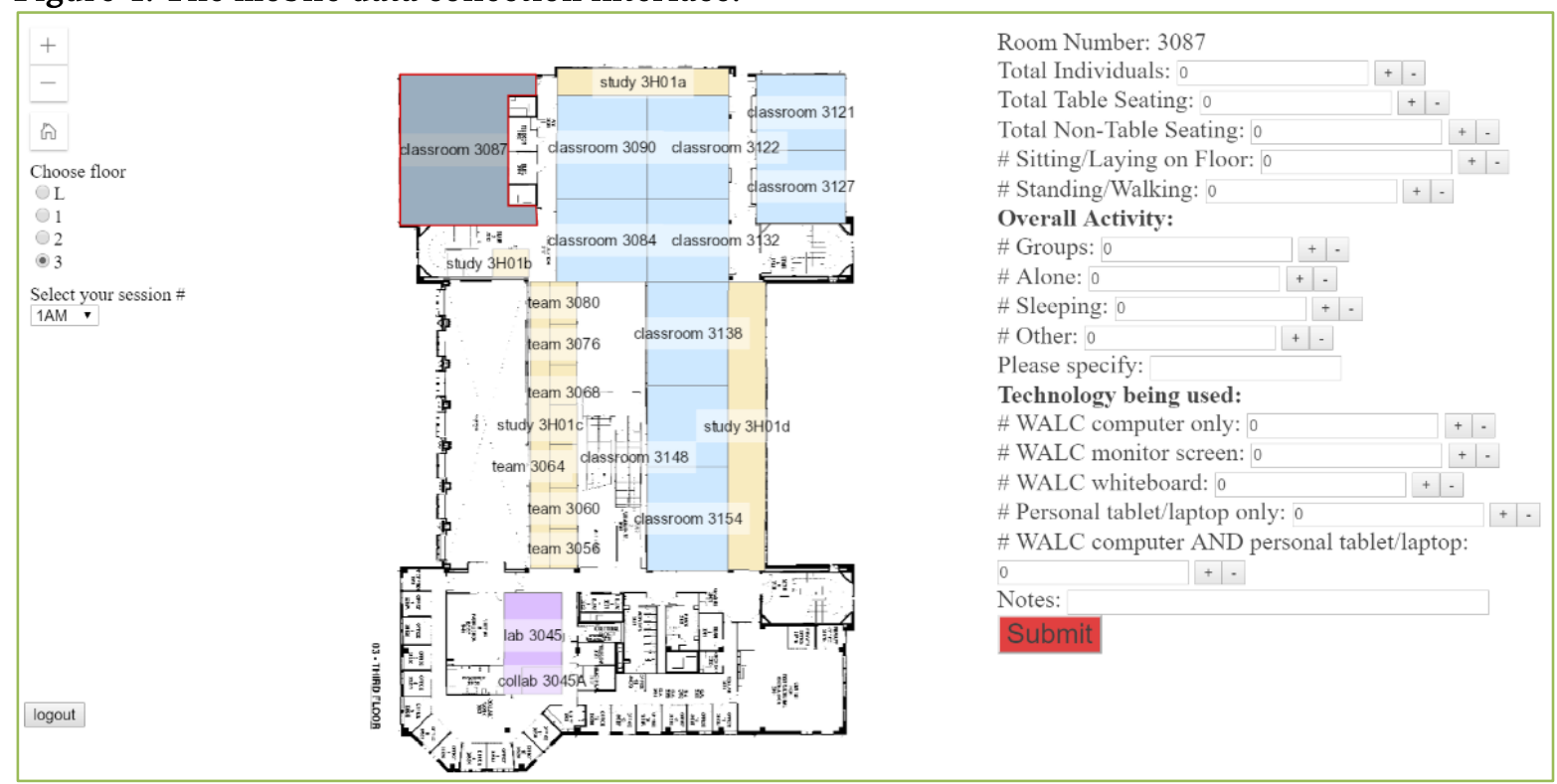

In the mobile data collection interface, the feature services were overlaid with the WALC's background floor plans. As shown in Figure 5, when a user clicks on any room or space from the feature map, this feature is highlighted and the data collection panel, or form, will appear. The user can then enter all observations for that room into the data collection form, as well as add free-text notes if needed. When entering numbers, the interface allows users to count by clicking the up/down arrows in each box, or to type in numbers directly. After all observations have been entered for a room, clicking the submit button transmits all input data, as well as the time/date stamp and user information, to the corresponding table in the geodatabase.

To avoid duplicated efforts when working as a team, a mechanism was designed to allow users to monitor each other's data collection activity. When observations for a room or space is submitted in a particular time period as determined by the session number, that space is then shaded on the map. Maps for all users collecting data during the same session number are synchronized and updated immediately as data are submitted. This mechanism can also help users track their data collection progress. When a user clicks on a shaded space, a pop-up message panel will provide details regarding the last submission for that space (Figure 5). 
Figure 5. Mobile data collection interface: rooms are shaded if the information has been submitted

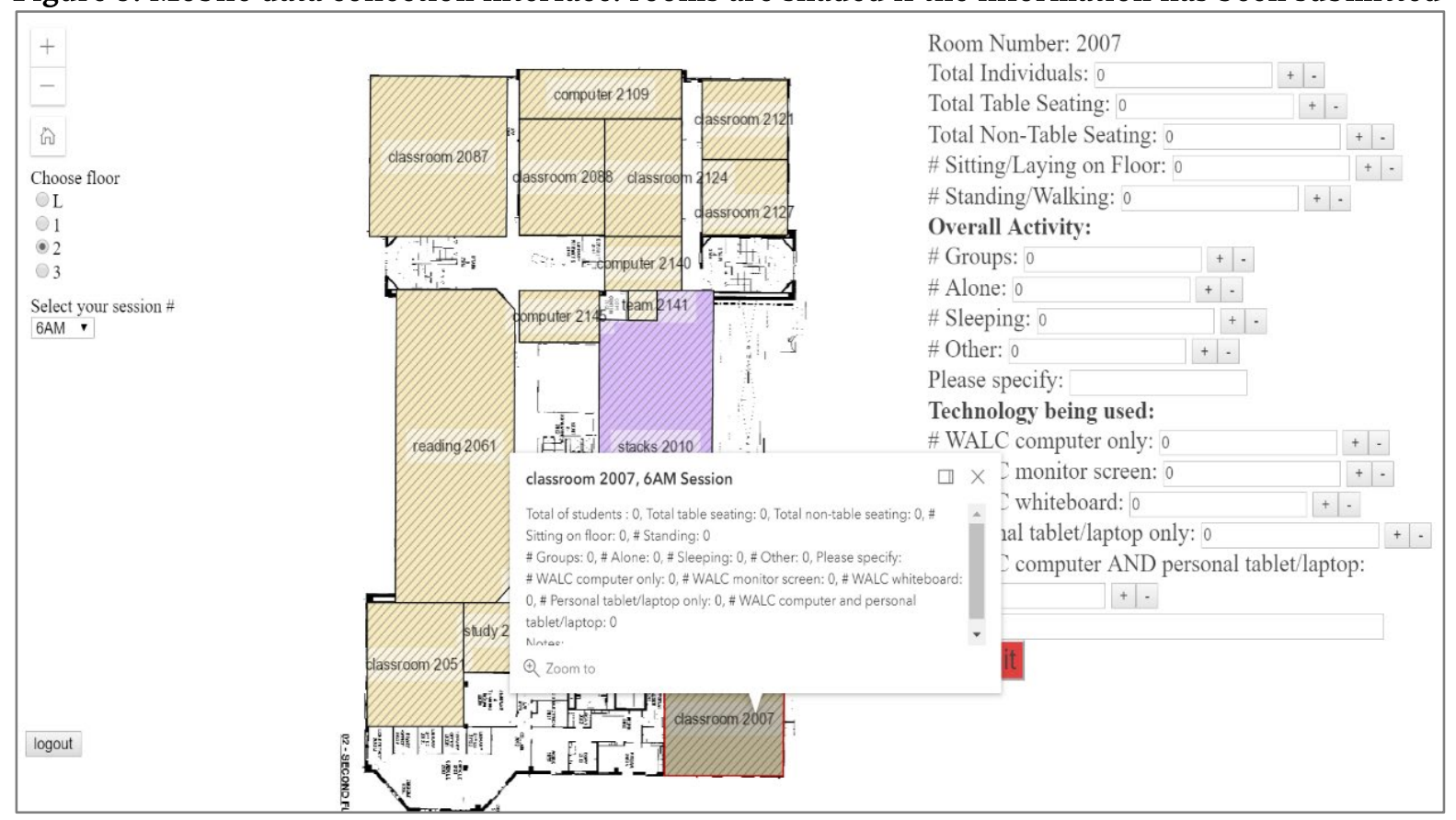

After designing the interface, it was determined that an efficient method would also be needed to communicate information to project members interested in reviewing results post-data-collection. Although all observations are saved to the geodatabase, generating a useful analysis of that information required additional software to connect to the geodatabase and an SQL query written to aggregate the data, as well as a good understanding of the database structure. To facilitate this process, a reporting feature was designed using the SQL Server Reporting Service from the database server to aggregate collected information and to generate scheduled reports. These reports are automatically saved to a designated folder on the departmental server at a prescribed time each morning, thus allowing results to be quickly and efficiently shared with the team.

\section{Conclusions and Limitations}

Findings to-date suggest the data-collection system is an effective tool for compiling library usage observations with minimal end-user training requirements. The system enables multiple staff members to collect consistent information in a reduced amount of time. Initial analyses have also helped to inform understanding of learning space usage statistics and the different learning activities occurring in the integrated areas, which will serve as a guideline for PUL's future service design.

As with any mobile application, this system is highly dependent on a strong wi-fi connection and a stable server environment. A data-submission failure could occur if observations are submitted in areas with weak or spotty Wi-Fi signals. Also, all server maintenance should be carefully scheduled around data collection activity to avoid connection interruptions.

\section{Future Research}

The PUL observation data collection system was initially designed for use in the WALC building, but the intent is to next expand the system to include all Purdue libraries. However, doing so will require changes to the mobile data collection interface to accommodate floorplans for different buildings, as well as to allow the user to choose a specific library. Future developments could also include making the system available as a library resource and extending its use to non-library locations as well.

-Copyright 2019 Karen R. Hum, Ningning Kong, Yue Li, and Nanette Andersson 


\section{Karen R. Hum, PhD}

Director of Assessment

Ningning Kong, PhD

Associate Professor, GIS Specialist

Yue Li

Geographic Information Systems (GIS) Analyst

Nanette Andersson, M.ARCH, AIA, NCARB, LEED AP, FMP

Director of Libraries Facilities

1. Beaudoin, et al.; Fried Foster, et al.

2. Casden and Davidson.

3. Godfrey and Stoddart.

4. See "Technical System Design," below.

5. ESRI.

\section{Bibliography}

Beaudoin, Diane, Craig Zywicki, Ada Uche, Michael Flierl, and Tomalee Doan. "Influence of Learning Space and Physical Design on Students' Interactive Learning." Purdue University Office of Institutional Assessment, 2016-2017, https://www.purdue.edu/oirae/documents/White_Papers/ALS\%20\%20Full.pdf

Casden, J., and B. Davidson. "The Suma Project: Integrating Observational Data Assessment into Space and Service Design.” Presented at the ACRL 2013 Cyber Zed Shed track, Indianapolis, IN, April 11, 2013. https://speakerdeck.com/bretdavidson/the-suma-project-integrating-observational-data-assessmentinto-space-and-service-design.

DEGW. "A Study of Trends in Pedagogy at Purdue: Analysis of the Impact of Changes in Pedagogy and Study Needs on Space Planning." Purdue e-Pubs. Office of the Provost Publications and Reports, 2012. http://docs.lib.purdue.edu/provost_pubs/2.

ESRI 2017. ArcGIS API for JavaScript 3.27. Redlands, CA: Environmental Systems Research Institute.

Fried Foster, Nancy, Teresa Balser, , Rae Lynn Boes, Dianna Deputy, William Ferrall, Michael Fosmire, Jeremy R. Garritano, Amanda Gill, Vicki Killion, Monica Kirkwood, Clarence Maybee, , Kristen Twardowski, Jane Yatcilla, and Tao Zhang. "Participatory Design of Purdue University's Active Learning Center Final Report.” Libraries Reports, 2013.

http://docs.lib.purdue.edu/libreports/1.

Godfrey, Bruce, and Rick Stoddart. "Managing In-Library Use Data: Putting a Web Geographic Information Systems Platform through its Paces." Information Technology and Libraries (Online) 37, no. 2 (2018): 3449. 